\title{
Statistical significance of displacements in heterogeneous control networks
}

\author{
Krzysztof Nowel, Waldemar Kamiński
}

\author{
University of Warmia and Mazury \\ Faculty of Geodesy on Land Management \\ Institute of Geodesy \\ 1 Oczapowskiego St., 10-719 Olsztyn, Poland \\ e-mail: krzysztof.nowel@uwm.edu.pl, \\ waldemar.kaminski@uwm.edu.pl
}

Received: 8 August 2013 / Accepted: 14 October 2013

\begin{abstract}
This paper proposes a modification of the classical process for evaluating the statistical significance of displacements in the case of heterogeneous (e.g. linear-angular) control networks established to deformation measurements and analysis. The basis for the proposed solution is the idea of local variance factors. The theoretical discussion was complemented with an example of its application on a simulated horizontal control network. The obtained results showed that the evaluation of the statistical significance of displacements in the case of heterogeneous control networks should be carried out using estimators of local variance factors.
\end{abstract}

Keywords: deformation analysis, statistical test, confidence ellipse, estimation of variance components

\section{Introduction}

As a result of processing two periodic measurements in a control network established to deformation measurements and analysis, we obtain the values of relative displacements of potential reference points and the values of displacements of controlled points in relation to actual reference points (absolute network) or the values of relative displacements of controlled points (relative network), e. g. (Chen, 1983; Chrzanowski and Chen, 1990; Caspary, 2000; Prószyński and Kwaśniak, 2006). These displacements are evaluated for significance with reference to the accuracy of the applied measurement method. The aim of the evaluation is to check if the determined value is the actual displacement of a given point or only the result of random measurement errors.

Control networks in which different geometric quantities are measured, with different geodetic equipment, in different atmospheric conditions or by different survey teams are called heterogeneous control networks. An example of such a network can be an linear-angular network established for deformation measurements and analysis. 
The classical evaluation of the significance of displacements for individual points in such networks is based on the a priori weight matrix of observations and the estimator of global variance factor e. g. (Chen, 1983; Chen et al., 1990; Caspary, 2000; Setan and Singh, 2001; Prószyński and Kwaśniak, 2006). This estimator is common for all adjusted observations and is, unfortunately, not very effective in the evaluation of weighting for heterogeneous measurement results. Evaluation of the significance of displacement based on incorrectly selected weight matrices of observations may lead to erroneous conclusions.

This paper proposes a modification of the classical process for evaluation of the significance of displacements in heterogeneous control networks intended for deformation surveys. The modification consists in introducing estimators of local variance factors, i.e. local estimators assigned to distinguished homogeneous groups of observations.

Generally, the problem of heterogeneous observations is well-known and has been dealt with in theory and practice. In literature we can find many methods of estimation of local variance factors, e. g. Henderson's estimation, MINQUE estimation, maximum likelihood estimation, Bayesian estimation or combination of maximum likelihood estimation and Bayesian estimation- generalized maximum likelihood estimation. However, these considerations concern only evaluating of statistical accuracy for adjustment results in geodetic networks (one epoch), while this problem has not been examined in the context of evaluating of statistical significance for displacements in control networks (many epochs). There is a lack of research showing the consequences of erroneous weighting for evaluating of statistical significance for displacements in control networks. Such detailed research has been carried out in this paper.

\section{Theoretical basis}

The aim of the process of evaluating the significance of displacement for the tested point $i$ is to check if these vectors are actual displacements or only the result of random measurement errors. Evaluating of statistical significance for displacements should be based on or supported by statistical null hypothesis

$$
H_{0}: E\left(\mathbf{d}_{i}\right)=0 \text {, }
$$

where

$$
\mathbf{d}_{i}=\mathbf{x}_{i_{(2)}}-\mathbf{x}_{i_{(1)}}
$$

is the displacement vector of the tested point $i, \mathbf{x}_{i_{(e)}}$ is the vector of coordinates of the tested point $i$ from $e$ th measurement epoch and $E(\cdot)$ is the statistical expectation. If the null hypothesis is accepted, this means that the displacement of the tested point $i$ is not significance and thus this point is identified as not displaced. If the null hypothesis is 
rejected, this means that the displacement of the tested point $i$ is significance and thus this point is identified as displaced. The null hypothesis can be accepted if following condition is fulfilled (local significance test)

$$
T_{i}=\frac{\mathbf{d}_{i}^{T} \mathbf{Q}_{\mathbf{d}_{i}}^{-1} \mathbf{d}_{i}}{u_{\mathbf{d}_{i}} \sigma_{0}^{2}}<F_{\alpha}\left(\alpha, u_{\mathbf{d}_{i}}, d f\right),
$$

where $\mathbf{Q}_{\mathbf{d}_{i}}$ is the cofactor matrix of the vector $\mathbf{d}_{i}, u_{\mathbf{d}_{i}}$ is dimension of the vector $\mathbf{d}_{i}, \sigma_{0}^{2}$ is the common global variance factor for two measurement epochs, $\alpha$ is significance level of the results, $d f$ is number of degrees of freedom in the control network from two measurement epochs, $F_{\alpha}$ is value read from Snedecor's $F$-distribution tables for a combination of degrees of freedom $f_{1}=u_{\mathrm{d}_{i}}$ and $f_{2}=d f$ and the assumed significance level $\alpha$.

The solution (3) can be used for 1-D, 2-D and 3-D networks. Moreover, another condition can be derived from the condition (3) for better interpretation of results

$$
\mathbf{d}_{i} \in E_{\alpha}=f\left(F_{\alpha}, \sigma_{0}^{2}, \mathbf{Q}_{\mathbf{d}_{i}}\right),
$$

where $E_{\alpha}$ is the confidence interval (1-D network) or the set of points formed by the confidence ellipse (2-D network), e. g. (Kamiński and Nowel, 2013) or the confidence ellipsoid (3-D network), e. g. (Cederholm, 2003). In this case, evaluation of significance consists in checking graphically whether the vector $\mathbf{d}_{i}$ does not exceed the confidence interval for determination of this vector (1-D network) or the confidence ellipse for determination of this vector (2-D network) or the confidence ellipsoid for determination of this vector (3-D network). If the conditions (3) or (4) are fulfilled, the displacement vector of point $i$ is considered insignificant. If this is not the case, this vector is found significant and point $i$ displaced. Of course, both solutions (3), (4) give the same results.

It should be necessarily noted that the evaluation of significance is of a probabilistic nature. Not finding the significance of the displacement vector of a tested point $i$ does not mean mathematical invariability of its position. This only means that it is found at the level of particular measurement errors, with particular probability $\gamma=1-\alpha$, that the given point has not been displaced.

\subsection{Classical evaluation of the significance of displacement vector}

After determination of the vector of displacement for all analyzed points in the control network, a common estimator of global variance factor for two measurement epochs is calculated e. g. (Chen, 1983; Chen et al., 1990; Caspary, 2000; Setan and Singh, 2001; Prószyński and Kwaśniak, 2006). In the theoretical model, which is the linearized form of the initial nonlinear relationships 


$$
\mathbf{A}_{(e)} \boldsymbol{\delta}_{\mathbf{x}_{(e)}}-\mathbf{v}_{(e)}=\mathbf{w}_{(e)}, \mathbf{w}_{(e)} \sim N\left[\mathbf{A}_{(e)} \boldsymbol{\delta}_{\mathbf{x}_{(e)}}, \mathbf{C}_{(e)}\right]
$$

where $\mathbf{A}_{(e)} \in \mathfrak{R}^{n \times u}$ is design matrix, $\boldsymbol{\delta}_{\mathrm{x}(e)} \in \mathfrak{R}^{u \times 1}$ is the vector of the corrections to the approximate coordinates for all analyzed points in the control network, $\mathbf{v}_{(e)} \in \mathfrak{R}^{u \times 1}$ is the vector of residuals, $\mathbf{w}_{(e)}=\mathbf{l}_{(e)}-\mathbf{l}_{(e)}^{0}, \mathbf{l}_{(e)} \in \mathfrak{R}^{n \times 1}$ is the vector of actual observations, $\mathbf{l}_{(e)}^{0} \in \mathfrak{R}^{n \times 1}$ is the vector of approximate observations, $\mathbf{C}_{(e)} \in \mathfrak{R}^{n \times n}$ is the covariance matrix of observations, $n$ is a number of observations and $u$ is number of unknowns, a common estimator of global variance factor for two measurement epochs is calculated on the basis of the formula

$$
\hat{\sigma}_{0}^{2}=\frac{d f_{(1)}\left(\hat{\sigma}_{0(1)}^{2}\right)+d f_{(2)}\left(\hat{\sigma}_{0(2)}^{2}\right)}{d f},
$$

with

$\hat{\sigma}_{0(e)}^{2}=\hat{\mathbf{v}}_{(e)}^{T} \mathbf{P}_{(e)} \hat{\mathbf{v}}_{(e)} / d f_{(e)}$ is the estimator of global variance factor,

$\hat{\mathbf{v}}_{(e)}=\mathbf{A}_{(e)} \hat{\boldsymbol{\delta}}_{\mathbf{x}(e)}-\left(\mathbf{l}_{(e)}-\mathbf{l}_{(e)}^{0}\right)$ is the estimator of the vector of residuals,

$\hat{\boldsymbol{\delta}}_{\mathbf{x}(e)}=\mathbf{N}_{(e)}^{-} \mathbf{A}_{(e)}^{T} \mathbf{P}_{(e)} \mathbf{w}_{(e)}$ is the estimator of the vector of the corrections to the approximate coordinates for all analyzed points in the control network,

$\hat{\mathbf{x}}_{(e)}=\mathbf{x}_{(e)}^{0}+\hat{\boldsymbol{\delta}}_{\mathbf{x}(e)}$ is the vector of adjusted coordinates for all analyzed points in the control network and

$d f=d f_{(1)}+d f_{(2)}$

$\mathbf{N}=\mathbf{A}_{(e)}^{T} \mathbf{P}_{(e)} \mathbf{A}_{(e)}$,

where $\mathbf{P}_{(e)} \in \mathfrak{R}^{n \times n}$ is weight matrix of observations, $\mathbf{x}_{(e)}^{0}$ is the vector of approximate coordinates for all analyzed points in the control network, $d f_{(e)}$ is number of degrees of freedom in the control network. For a matrix $\mathbf{N}_{(e)}$ used $g$-inverse. This is due to the fact that the control networks are free networks. In this case, the matrix $\mathbf{A}_{(e)}$ is the matrix of the columnarly incomplete rank, which is why the matrix $\mathbf{N}_{(e)}$ is singular and there is no ordinary (classical) inverse $(\cdot)^{-1}$. From the point of view of geodesy, we have a datum defect problem for the estimator $\hat{\boldsymbol{\delta}}_{\mathbf{x}(e)}$ and thus for the estimator $\hat{\mathbf{d}}$. To solve this problem, the datum is defined on a group of stable points. In fact, we put on these points, certain conditions (constraints), e. g. the condition of constancy for this points or the condition of minimum trace of cofactor matrix for this points. This problem is well-known. More information can be found in papers: e. g. (Prószyński, 1986; Chen et al., 1990; Prószyński and Kwaśniak, 2006).

The estimator $\hat{\sigma}_{0(e)}^{2}$ is common for all adjusted observations e.g. for linear and angular observations in linear-angular networks. 
Cofactor matrix of the estimator of the displacement vector can be presented in the following form

$$
\mathbf{Q}_{\hat{\mathbf{d}}}=\mathbf{Q}_{\hat{\mathbf{x}}_{(1)}}+\mathbf{Q}_{\hat{\mathbf{x}}_{(2)}}=\left[\begin{array}{cccc}
\mathbf{Q}_{\hat{\mathbf{d}}_{1}} & \mathbf{Q}_{\hat{\mathbf{d}}_{1}, \hat{\mathbf{d}}_{2}} & \cdots & \mathbf{Q}_{\hat{\mathbf{d}}_{1}, \hat{\mathbf{d}}_{z}} \\
\mathbf{Q}_{\hat{\mathbf{d}}_{2}, \hat{\mathbf{d}}_{1}} & \mathbf{Q}_{\hat{\mathbf{d}}_{2}} & \cdots & \mathbf{Q}_{\hat{\mathbf{d}}_{2}, \hat{\mathbf{d}}_{z}} \\
\vdots & \vdots & \ddots & \vdots \\
\mathbf{Q}_{\hat{\mathbf{d}}_{z}, \hat{\mathbf{d}}_{1}} & \mathbf{Q}_{\hat{\mathbf{d}}_{z}, \hat{\mathbf{d}}_{2}} & \cdots & \mathbf{Q}_{\hat{\mathbf{d}}_{z}}
\end{array}\right],
$$

with

$\mathbf{Q}_{\hat{\mathbf{x}}_{(e)}}=\mathbf{N}_{(e)}^{-} \in \Re^{u \times u}$ is the cofactor matrix of the vector $\hat{\mathbf{x}}_{(e)}$,

$\mathbf{Q}_{\hat{\mathbf{d}}_{i}}=\left[\begin{array}{cc}Q_{\hat{d}_{x_{i}}} & Q_{\hat{d}_{i} \hat{d}_{y_{i}}} \\ Q_{\hat{d} y_{i} \hat{d}_{i}} & Q_{\hat{d} \hat{y}_{i}}\end{array}\right]$ (for 2-D network) is the cofactor matrix of the vector $\hat{\mathbf{d}}_{i}$.

After assuming the significance level $\alpha$ i.e. a quantity such that

$$
P\left\{\hat{\mathbf{d}}_{i} \notin E_{\alpha}\right\}=\alpha
$$

evaluation of the significance of the vector $\hat{\mathbf{d}}_{i}$ is conducted. Based on the estimator $\hat{\sigma}_{0}^{2}$, the matrix $\mathbf{Q}_{\hat{\mathbf{d}}_{i}}$ and the assumed significance level $\alpha$ the parameters of a confidence ellipse (for 2-D network) are determined. These parameters are the semi-axes of the ellipse (9) and the twist angle of the ellipse, i.e. the angle included between the semimajor axis and the $\mathrm{X}$-axis of the determined coordinate system (10)

$$
\begin{aligned}
& a_{i}=\hat{\sigma}_{0} \sqrt{2 \lambda_{1} F_{\alpha}}, \\
& b_{i}=\hat{\sigma}_{0} \sqrt{2 \lambda_{2} F_{\alpha}} \text {, } \\
& \phi_{i}=\frac{1}{2} \operatorname{arctg} \frac{2 Q_{\hat{d}_{i} \hat{d}_{y_{i}}}}{Q_{\hat{d x_{i}}}-Q_{\hat{d}_{y_{i}}}},
\end{aligned}
$$

with

$$
\begin{gathered}
\lambda_{1}=\left(Q_{\hat{d} x_{i}}+Q_{\hat{d} y_{i}}+\Delta_{i}\right) / 2, \\
\lambda_{2}=\left(Q_{\hat{d} x_{i}}+Q_{\hat{d} y_{i}}-\Delta_{i}\right) / 2, \\
\Delta_{i}=\sqrt{\left(Q_{\hat{d} x_{i}}-Q_{\hat{d} y_{i}}\right)^{2}+4 Q_{\hat{d} x_{i} \hat{d} y_{i}}^{2}},
\end{gathered}
$$

where $F_{\alpha}$ is value read from Snedecor's $F$-distribution tables for a combination of degrees freedom $f_{1}=u, f_{2}=n-u$ and the assumed significance level $\alpha$. 
The condition (4) is most often checked by the graphical method. The confidence ellipse $E_{\alpha}$ (for 2-D network) is superimposed on vector $\hat{\mathbf{d}}_{i}$ in such a way that its centre coincides with the origin of this vector (Fig. 1)

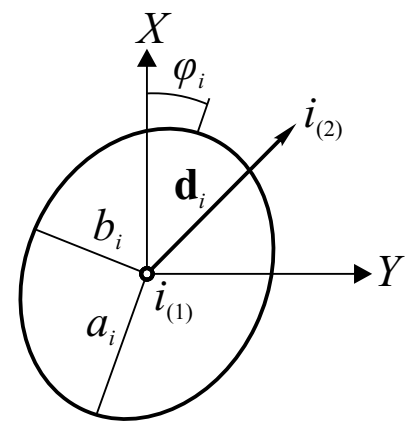

Fig. 1. Displacement vector and confidence ellipse

If vector $\hat{\mathbf{d}}_{i}$ lies within the confidence ellipse, the displacement is considered insignificant. In the opposite case, it is assumed that at the level of the specified measurement errors and the assumed significance level of the results, the tested point $i$ was displaced.

The procedure presented above is classical and almost always applied in practice.

\subsection{Proposal of modification for classical evaluation of the significance of displacement vector}

The presented algorithm is based on estimation of local variance factors, i.e. local $\sigma_{0 l}^{2}$ assigned to distinguished homogeneous groups of observations $(l=1,2, \ldots, r$ - designation of homogeneous groups of observations). However, it should be noted that the criterion for dividing a set of observations into groups does not necessarily have to result from the type of performed measurements, but also from other features, e.g. the type of instruments used, the time of vector measurement using GNSS technology, etc. Henderson's method (Henderson, 1953; Wiśniewski, 1989; 1990) was adapted in the algorithm. This method is simpler and more natural than other methods of estimation of local variance factors.

The original Henderson's system of equations, adopted to model (5) can be presented in the following simple form (Henderson, 1953)

$$
E\left(\mathbf{w}^{T} \mathbf{h}_{l} \mathbf{w}\right)=\mathbf{w}^{T} \mathbf{h}_{l} \mathbf{w},
$$

for each $l=1, \ldots, r$, where $\mathbf{h}_{l}$ is some unknown matrix. On the basis of the system of equations (14) we obtain 


$$
\operatorname{Tr}\left(\mathbf{h}_{l} \mathbf{C}\right)+E(\mathbf{w})^{T} \mathbf{h}_{l} E(\mathbf{w})=\mathbf{w}^{T} \mathbf{h}_{l} \mathbf{w},
$$

where $\operatorname{Tr}(\cdot)^{-1}$ denotes a trace of the matrix, $\mathbf{C}=\mathbf{G P}^{-1}$ and $\mathbf{G}=\operatorname{diag}\left(\ldots, \sigma_{0 l}^{2}, \ldots\right)$. Because $E(\mathbf{w})=\mathbf{A} \boldsymbol{\delta}_{\mathbf{x}}$ (5) then

$$
\operatorname{Tr}\left(\mathbf{h}_{l} \mathbf{C}\right)+\boldsymbol{\delta}_{\mathbf{x}}^{T} \mathbf{A}^{T} \mathbf{h}_{l} \mathbf{A} \boldsymbol{\delta}_{\mathbf{x}}=\mathbf{w}^{T} \mathbf{h}_{l} \mathbf{w} .
$$

In the Henderson's estimation, we have to find $r$ matrix $\mathbf{h}_{l}$ which fulfill the condition

$$
\boldsymbol{\delta}_{\mathbf{x}}^{T} \mathbf{A}^{T} \mathbf{h}_{l} \mathbf{A} \boldsymbol{\delta}_{\mathbf{x}}=\mathbf{0} \Rightarrow \mathbf{A}^{T} \mathbf{h}_{l} \mathbf{A}=\mathbf{0},
$$

for each $l=1, \ldots, r$ and then we have to solve the equations system

$$
\operatorname{Tr}\left(\mathbf{h}_{l} \mathbf{C}\right)=\mathbf{w}^{T} \mathbf{h}_{l} \mathbf{w}
$$

with respect to the unknown local variance factors $\ldots, \sigma_{0 l}^{2}, \ldots$. The condition (17) is very important here. These condition means, that the determined further estimators will be unbiased.

In geodetic problems the variance factor is estimated on the basis of quadratic form $\hat{\mathbf{v}}^{T} \mathbf{P} \hat{\mathbf{v}}$. Analogy, the local variance factors should be estimated on the basis of local quadratic forms $\hat{\mathbf{v}}_{l}^{T} \mathbf{P}_{l} \hat{\mathbf{v}}_{l}$. Thus, we see that in order to adopt the Henderson's method for the geodetic problems, the quadratic forms $\hat{\mathbf{v}}_{l}^{T} \mathbf{P}_{l} \hat{\mathbf{v}}_{l}$ should be brought to the Henderson's quadratic forms $\mathbf{w}^{T} \mathbf{h}_{l} \mathbf{w}$. In fact, we have to determine the matrix $\mathbf{h}_{l}$, which fulfill the condition of the unbiased estimators $\ldots, \sigma_{0 l}^{2}, \ldots$ (17). For this purpose, we write

$$
\hat{\mathbf{v}}_{l}=\mathbf{A}_{l} \hat{\boldsymbol{\delta}}_{\mathbf{x}}-\mathbf{w}_{l}=\mathbf{A}_{l} \mathbf{N}^{-} \mathbf{A}^{T} \mathbf{P} \mathbf{w}-\mathbf{w}_{l}=-\mathbf{F}_{l} \mathbf{w},
$$

where

$$
\begin{aligned}
& \mathbf{A}^{T} \in \mathfrak{R}^{u \times n}=\left[\ldots, \mathbf{A}_{l}^{T} \in \mathfrak{R}^{u \times n_{l}}, \ldots\right], \\
& \mathbf{P} \in \mathfrak{R}^{n \times n}=\operatorname{diag}\left(\ldots, \mathbf{P}_{l} \in \mathfrak{R}^{n_{l} \times n_{l}}, \ldots\right), \\
& \mathbf{F}_{l} \in \mathfrak{R}^{n_{l} \times n}=\mathbf{I}_{0 l}-\mathbf{A}_{l} \mathbf{N}^{-} \mathbf{A}^{T} \mathbf{P},
\end{aligned}
$$

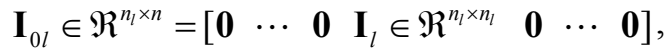

$\mathbf{I}_{l}$ is the identity matrix and $n_{l}$ is the number of observations for the $l$ th homogeneous group.

Further, on the basis of the equations system (19) we obtain 


$$
\hat{\mathbf{v}}_{l}^{T} \mathbf{P}_{l} \hat{\mathbf{v}}_{l}=\left(\mathbf{F}_{l} \mathbf{w}\right)^{T} \mathbf{P}_{l} \mathbf{F}_{l} \mathbf{w}=\mathbf{w}^{T} \mathbf{h}_{l} \mathbf{w},
$$

where

$$
\mathbf{h}_{l}=\mathbf{F}_{l}^{T} \mathbf{P}_{l} \mathbf{F}_{l} .
$$

After substitution of the matrix $\mathbf{h}_{l}$ (21) into the system of equations (16), we obtain

$$
\operatorname{Tr}\left(\mathbf{h}_{l} \mathbf{C}\right)+\boldsymbol{\delta}_{\mathbf{x}}^{T} \mathbf{A}^{T} \mathbf{F}_{l}^{T} \mathbf{P}_{l} \mathbf{F}_{l} \mathbf{A} \boldsymbol{\delta}_{\mathbf{x}}=\mathbf{w}^{T} \mathbf{h}_{l} \mathbf{w} .
$$

The condition of the unbiased estimators (17) is fulfilled by assumed matrix $\mathbf{h}_{l}$, because

$$
\mathbf{A}^{T} \mathbf{F}_{l}^{T}=\mathbf{A}^{T}\left(\mathbf{I}_{0 l}^{T}-\mathbf{P} \mathbf{A} \mathbf{N}^{-} \mathbf{A}_{l}^{T}\right)=\mathbf{A}_{l}^{T}-\mathbf{A}^{T} \mathbf{P} \mathbf{A} \mathbf{N}^{-} \mathbf{A}_{l}^{T}=\mathbf{0} .
$$

Determining the left side of the equations system we obtain (Appendix 1)

$$
\operatorname{Tr}\left(\mathbf{h}_{l} \mathbf{C}\right)=q_{l} \sigma_{0 l}^{2}+\sum_{k=1}^{l-1} c_{l k} \sigma_{0 k}^{2}+\sum_{k=l+1}^{r} c_{l k} \sigma_{0 k}^{2}
$$

where

$$
\begin{aligned}
& q_{l}=n_{l}-2 \operatorname{Tr}\left(\mathbf{N}_{l} \mathbf{N}^{-}\right)+\operatorname{Tr}\left(\mathbf{N}_{l} \mathbf{N}^{-} \mathbf{N}_{l} \mathbf{N}^{-}\right), \\
& c_{l k}=\operatorname{Tr}\left(\mathbf{N}_{l} \mathbf{N}^{-} \mathbf{N}_{k} \mathbf{N}^{-}\right), \\
& \mathbf{N}_{l}=\mathbf{A}_{l}^{T} \mathbf{P}_{l} \mathbf{A}_{l}, \\
& \mathbf{N}_{k}=\mathbf{A}_{k}^{T} \mathbf{P}_{k} \mathbf{A}_{k} \text { and }
\end{aligned}
$$

$l, k=1, \ldots, r$ is the designation of homogeneous groups of observations.

Now, the Henderson's equations system (18) can be written in the form

$$
q_{l} \sigma_{0 l}^{2}+\sum_{k=1}^{l-1} c_{l k} \sigma_{0 k}^{2}+\sum_{k=l+1}^{r} c_{l k} \sigma_{0 k}^{2}=\mathbf{w}^{T} \mathbf{h}_{l} \mathbf{w} .
$$

Finally, on the basis of the equations system (25), the equations system enabling estimation of local variance factors can be presented in the following form

$$
\left[\begin{array}{c}
\hat{\sigma}_{01}^{2} \\
\hat{\sigma}_{02}^{2} \\
\vdots \\
\hat{\sigma}_{0 r}^{2}
\end{array}\right]=\left[\begin{array}{cccc}
q_{l} & c_{12} & \cdots & c_{1 r} \\
c_{21} & q_{2} & \cdots & c_{2 r} \\
\vdots & \vdots & \ddots & \vdots \\
c_{r 1} & c_{r 2} & \cdots & q_{r}
\end{array}\right]^{-1}\left[\begin{array}{c}
\mathbf{w}^{T} \mathbf{h}_{1} \mathbf{w} \\
\mathbf{w}^{T} \mathbf{h}_{2} \mathbf{w} \\
\vdots \\
\mathbf{w}^{T} \mathbf{h}_{r} \mathbf{w}
\end{array}\right] .
$$


Estimators of local variance factors (26) are calculated independently for two measurement epochs $(e=1,2)$. The calculations are carried out in an iterative cycle with the following formula

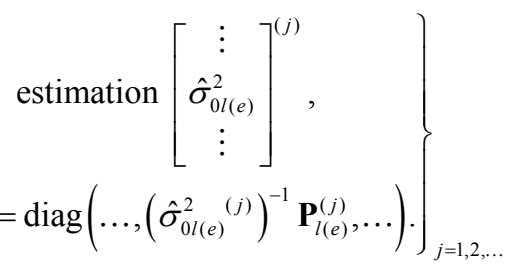

In subsequent iterative steps, new matrices $\mathbf{P}_{(e)}$ are obtained. After each next step, the values of this matrix are closer to the "real" values. The process (27) is finished at the moment when the following condition is fulfilled

$$
\hat{\sigma}_{0(e)}^{2} \cong \ldots \cong \hat{\sigma}_{0 l(e)}^{2} \cong \ldots \cong 1
$$

(within the limits of the assumed solution precision).

Sufficiently accurately estimated matrices $\mathbf{P}_{(e)}$ are obtained from the last iterative step. On their basis, the cofactor matrix of the displacement vector (7) is determined.

After assuming the significance level $\alpha$, the parameters of the confidence ellipse can already be determined for the displacement vector. Because $\hat{\sigma}_{0}^{2} \cong 1$, the parameters of a confidence ellipse will now assume the form

$$
\left.\begin{array}{l}
a_{i}=\sqrt{2 \lambda_{1} F_{\alpha}}, \\
b_{i}=\sqrt{2 \lambda_{2} F_{\alpha}},
\end{array}\right\}
$$

and (10).

Afterwards, we proceed the same as in the case of classical evaluation of significance.

\section{Numerical test}

The practical properties of the proposed solution were analyzed based on the example of a simulated linear-angular network. The analyzed network consists of two stable reference points A, B and one controlled point $i$ (absolute network), (Fig. 2). By way of simplification, a rigid reference system was assumed, i.e. with zero values of displacements at points A, B. 


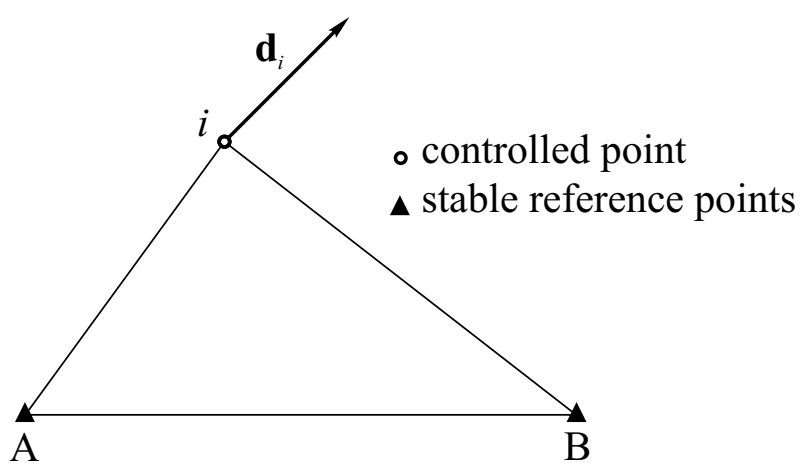

Fig. 2. Control network

In the first place, theoretical coordinates of points $\mathrm{A}, \mathrm{B}, i$ were assumed in the initial and actual measurements. Displacement was simulated for point $i$, adding in the actual measurement $(e=2)$ the value of $0.010 \mathrm{~m}$ to the coordinates $\mathrm{X}$, Y. The theoretical coordinates of points A, B, $i$ have been compiled in Table 1 .

Table 1. Theoretical coordinates of points

\begin{tabular}{|c|c|c|c|c|c|}
\hline \multicolumn{3}{|c|}{ Initial measurement $(e=1)$} & \multicolumn{3}{|c|}{ Actual measurement $(e=2)$} \\
\hline point & $\mathrm{X}[\mathrm{m}]$ & $\mathrm{Y}[\mathrm{m}]$ & point & $\mathrm{X}[\mathrm{m}]$ & $\mathrm{Y}[\mathrm{m}]$ \\
\hline $\mathrm{A}$ & 0.000 & 0.000 & $\mathrm{~A}$ & 0.000 & 0.000 \\
\hline $\mathrm{B}$ & 0.000 & 100.000 & $\mathrm{~B}$ & 0.000 & 100.000 \\
\hline$i$ & 100.000 & 30.000 & $i$ & 100.010 & 30.010 \\
\hline
\end{tabular}

Next, the results of two periodic measurements, randomly disturbed with measurement errors (Table 2), were generated based on theoretical coordinates (Table 1). Normal distribution of "real" measurement errors for angles and distances was assumed with standard deviations

$$
\begin{aligned}
\sigma_{\beta} & =14^{\mathrm{cc}}, \\
\sigma_{s} & =0.006 \mathrm{~m}
\end{aligned}
$$

and the assumed significance level $\alpha=0.05$. In order to simplify the calculations, observations in both measurement epochs were disturbed with exactly the same errors. 
Table 2. Simulated results of the measurements

\begin{tabular}{|c|c|c|c|c|c|c|c|}
\hline \multicolumn{4}{|c|}{ Initial measurement $(e=1)$} & \multicolumn{4}{|c|}{ Actual measurement $(e=2)$} \\
\hline \multicolumn{3}{|c|}{ observation code } & \multirow{2}{*}{ observations } & \multicolumn{3}{|c|}{ observation code } & \multirow{2}{*}{ observations } \\
\hline$c$ & $l$ & $r$ & & $c$ & $l$ & $r$ & \\
\hline A & $i$ & B & $81^{\mathrm{g}} 44^{\mathrm{c}} 66^{\mathrm{cc}}$ & A & $i$ & $\mathrm{~B}$ & $81^{\mathrm{g}} 44^{\mathrm{c}} 25^{\mathrm{cc}}$ \\
\hline B & A & $i$ & $61^{\mathrm{g}} 12^{\mathrm{c}} 15^{\mathrm{cc}}$ & B & A & $i$ & $61^{\mathrm{g}} 12^{\mathrm{c}} 87^{\mathrm{cc}}$ \\
\hline$i$ & B & A & $57^{\mathrm{g}} 43^{\mathrm{c}} 45^{\mathrm{cc}}$ & $\mathrm{i}$ & B & A & $57^{\mathrm{g}} 43^{\mathrm{c}} 14^{\mathrm{cc}}$ \\
\hline- & A & $i$ & $104.409 \mathrm{~m}$ & - & A & $i$ & $104.422 \mathrm{~m}$ \\
\hline- & $i$ & $\mathrm{~B}$ & $122.061 \mathrm{~m}$ & - & $i$ & $\mathrm{~B}$ & $122.063 \mathrm{~m}$ \\
\hline
\end{tabular}

Adjustment was carried out by the least squares method separately for two variants differing in the start values of a priori mean errors of measurement results assumed for calculations. These values were the basis for determining the weight matrix.

Variant 1 assumed

$$
\begin{aligned}
& m_{\beta}=10^{\mathrm{cc}}, \\
& m_{s}=0.010 \mathrm{~m}
\end{aligned}
$$

while variant 2 assumed

$$
\begin{aligned}
& m_{\beta}=20^{\mathrm{cc}}, \\
& m_{s}=0.005 \mathrm{~m} .
\end{aligned}
$$

Since standard deviations are known in this example, it can be said that in the first variant the value of the mean error of angle measurement was underestimated and the value of the mean error of side measurement overestimated. The opposite simulation was carried out in the second variant.

The displacement vector of point $i$ was determined by the method of coordinate differences. In the first and second measurement epochs, observations were disturbed with the same errors, which is why values of the displacement vector equal to assumed theoretical values were obtained in both variants.

In variant 1 we obtained $[\mathrm{m}]$

$$
\mathbf{d}_{i}=\hat{\mathbf{x}}_{i_{(2)}}-\hat{\mathbf{x}}_{i_{(1)}}=[0.010,0.010]^{T},
$$

where

$$
\hat{\mathbf{x}}_{i_{(1)}}=[100.002,30.000]^{T}, \hat{\mathbf{x}}_{i_{(2)}}=[100.012,30.010]^{T},
$$


while in variant $2[\mathrm{~m}]$

$$
\mathbf{d}_{i}=\hat{\mathbf{x}}_{i_{(2)}}-\hat{\mathbf{x}}_{i_{(1)}}=[0.010,0.010]^{T},
$$

where

$\hat{\mathbf{x}}_{i_{(1)}}=[100.002,30.001]^{T}, \hat{\mathbf{x}}_{i_{(2)}}=[100.012,30.011]^{T}$.

The estimators of global variance factors were then calculated and implementing the iterative cycle (27) estimators of local variance factors. In addition, the values of a priori mean errors of measurement results were determined on the basis of matrices $\mathbf{P}_{(e)}$ assumed for adjustment in individual iterative steps. The obtained results are presented in Table 3 and Table 4.

Table 3. Estimators of global and local variance factors

\begin{tabular}{|c|c|c|c|}
\hline \multirow{2}{*}{$\left.\begin{array}{c}\text { Iteration } \\
j\end{array}\right)$} & global & \multicolumn{2}{|c|}{ local } \\
\cline { 2 - 4 } & $\hat{\sigma}_{0(1)}^{2}, \hat{\sigma}_{0(2)}^{2}$ & $\hat{\sigma}_{0 \beta(1)}^{2}, \hat{\sigma}_{0 s(1)}^{2}$ & $\hat{\sigma}_{0 \beta(2)}^{2}, \hat{\sigma}_{0 s(2)}^{2}$ \\
\hline \multicolumn{4}{|c|}{ Variant 1} \\
\hline 1 & $0.96,0.99$ & $2.24,0.26$ & $2.24,0.32$ \\
\hline 2 & - & $0.74,1.33$ & $0.78,1.27$ \\
\hline 3 & - & $1.22,0.88$ & $1.17,0.91$ \\
\hline 4 & - & $0.92,1.07$ & $0.94,1.05$ \\
\hline 5 & - & $1.05,0.97$ & $1.03,0.98$ \\
\hline 6 & - & $0.98,1.02$ & $0.99,1.01$ \\
\hline & - & Variant 2 & $0.14,2.01$ \\
\hline 1 & $0.92,1.06$ & $0.21,1.67$ & $4.05,0.64$ \\
\hline 2 & - & $2.69,0.66$ & $0.78,1.26$ \\
\hline 3 & - & $0.78,1.25$ & $1.16,0.91$ \\
\hline 4 & - & $1.17,0.90$ & $0.94,1.04$ \\
\hline 5 & - & $0.93,1.05$ & $1.03,0.98$ \\
\hline 6 & - & $1.04,0.97$ & \\
\hline & & & \\
\hline
\end{tabular}


Table 4. A priori mean errors of measurement results

\begin{tabular}{|c|c|c|}
\hline \multirow{2}{*}{$\begin{array}{c}\text { Iteration } \\
(j)\end{array}$} & \multicolumn{2}{|c|}{$\begin{array}{c}\text { A priori mean errors of measurement } \\
\text { results assumed for adjustment }\end{array}$} \\
\cline { 2 - 3 } & $\left.m_{\beta(1)}{ }^{\mathrm{c}}\right], m_{s(1)}[\mathrm{m}]$ & $m_{\beta(2)}\left[{ }^{\mathrm{c}}\right], m_{s(2)}[\mathrm{m}]$ \\
\hline \multicolumn{3}{|c|}{ Variant 1} \\
\hline 1 & $10.0,0.010$ & $10.0,0.010$ \\
\hline 2 & $15.0,0.0051$ & $15.0,0.0057$ \\
\hline 3 & $12.9,0.0059$ & $13.2,0.0064$ \\
\hline 4 & $14.2,0.0056$ & $14.2,0.0061$ \\
\hline 5 & $13.7,0.0058$ & $13.8,0.0062$ \\
\hline 6 & $14.0,0.0057$ & $14.0,0.0062$ \\
\hline & & Variant 2 \\
\hline 1 & $20.0,0.005$ & $20.0,0.005$ \\
\hline 2 & $9.1,0.0065$ & $7.4,0.0071$ \\
\hline 3 & $14.9,0.0053$ & $15.0,0.0057$ \\
\hline 4 & $13.1,0.0059$ & $13.2,0.0064$ \\
\hline 5 & $14.2,0.0056$ & $14.2,0.0061$ \\
\hline 6 & $13.7,0.0057$ & $13.8,0.0062$ \\
\hline
\end{tabular}

The presented values show that estimators of global variance factors did not detect any irregularities in the weighting of the observations $\left(\hat{\sigma}_{0(e)}^{2} \cong 1\right)$, (Table 3$)$. The matrix $\mathbf{P}_{(e)}$ based on wrongly selected a priori mean errors of measurement results will therefore be falsified in both variants. Meanwhile, estimators of local variance factors rightly recognized these irregularities $\left(\hat{\sigma}_{0 \beta(e)}^{2}{ }^{(1)}, \hat{\sigma}_{0 s(e)}^{2}{ }^{(1)} \neq 1\right)$, (Table 3). After each iterative step a priori mean errors of measurement results were closer to actual values (Table 4). The computational process was stopped in the sixth iteration.

An erroneous matrix $\mathbf{P}_{(e)}$ may cause erroneous evaluation of the significance of the displacement vector. In order to confirm the above statement, the parameters of confidence ellipses for determination of the displacement vector were calculated on the basis of the estimation results for global and local variance factors. The significance level $\alpha=0.05$ was assumed for the calculations.

In analysing the results of calculations presented in Table 5, it can be seen that in both variants the parameters of the confidence ellipse based on the estimation results for global variance factors differ from actual values, determined on the basis of the estimation results for local variance factors. 
Table 5. Parameters of confidence ellipses

\begin{tabular}{|c|c|c|c|}
\hline \multirow{2}{*}{$\begin{array}{c}\text { Estimators of variance } \\
\text { factors }\end{array}$} & \multicolumn{3}{|c|}{ Parameters of confidence ellipses } \\
\cline { 2 - 4 } & $a_{i}[\mathrm{~m}]$ & $b_{i}[\mathrm{~m}]$ & $\phi_{i}[\mathrm{~g}]$ \\
\hline \multicolumn{4}{|c|}{ Variant 1} \\
\hline global & 0.010 & 0.008 & 15.5210 \\
\hline local & 0.013 & 0.011 & 22.8502 \\
\hline \multicolumn{4}{|c|}{ Variant 2} \\
\hline global & 0.017 & 0.015 & 47.0624 \\
\hline local & 0.013 & 0.011 & 22.2765 \\
\hline
\end{tabular}

Confidence ellipse regions based on global and estimators of local variance factors have been compared graphically below (Fig. 3, Fig. 4).

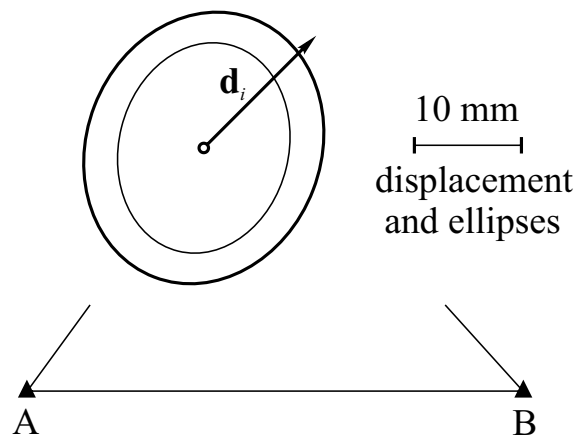

Fig. 3. Confidence ellipses for determination of the displacement vector of point $i$ using estimators of global (normal line) and local (bold line) variance factors - variant 1

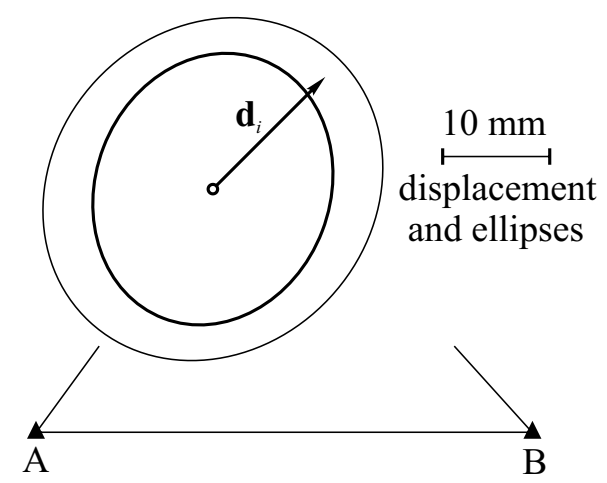

Fig. 4. Confidence ellipses for determination of the displacement vector of point $i$ using estimators of global (normal line) and local (bold line) variance factors - variant 2 
On the basis of the results shown in Fig. 3 and Fig. 4, we find that the presented confidence regions differ in both variants. In addition, in variant 2 , in the case of the confidence ellipse based on the estimation results for global variance factors, the displacement vector of point $i$ was interpreted as insignificant. Meanwhile, the confidence ellipse based on the estimation results for local variance factors indicates that the displacement of point $i$ is significant. The obtained results support the thesis formulated earlier that using estimators of global variance factors may lead in some cases to erroneous evaluation of the significance of displacement.

\section{Conclusions}

Geodetic methods are most often used for determination of minor displacements, with quantities slightly exceeding errors in their determination. The presented paper proves that classical evaluation of the significance of such displacements may lead to false conclusions in the case of heterogeneous control networks. The research carried out in this paper allows the authors to formulate the following recommendations:

i) the estimator of global (classical) variance factor can be applied only for evaluation of the significance of displacements in homogeneous control networks,

ii) evaluation of the significance of displacements in the case of heterogeneous (e.g. linear-angular) control networks should be carried out using estimators of local variance factors,

iii) in the case of displacements with quantities considerably exceeding the size of the confidence region, the issue of the variance factor is of no importance in evaluation of the significance of displacements.

\section{Acknowledgments}

The present study has been carried out as part of the statutory research No. 528-03020806 conducted at the Institute of Geodesy of the University of Warmia and Mazury in Olsztyn. The authors would like to thank reviewers for valuable remarks.

\section{References}

Caspary, W. F. (2000). Concepts of Network and Deformation Analysis. The University of New South Wales. Kensington, Australia.

Cederholm, P. (2003). Deformation Analysis Using Confidence Ellipsoids. Survey Review. Vol. 37, 31-45.

Chen, Y. Q. (1983) Analysis of Deformation Surveys - A Generalized Method. Technical Report No. 94, University of New Brunswick, Fredericton.

Chen, Y. Q., Chrzanowski A. \& Secord J. M. (1990). A strategy for the analysis of the stability of reference points in deformation surveys. CISM JOURNAL ACSGG., 44, 141-149.

Chrzanowski, A. \& Chen Y. Q. (1990). Deformation Monitoring, Analysis and Prediction-Status Report. FIG XIX Congress, Helsinki, Vol. 6, 83-97. 
Henderson, C. R. (1953). Estimation of Variance and Covariance Components. Biometrics., 9, 226-252.

Kamiński, W. \& Nowel K. (2013). Local variance factors in deformation analysis of non homogenous monitoring networks. Survey Review., 45, 44-50.

Prószyński, W. \& Kwaśniak M. (2006). Podstawy geodezyjnego wyznaczania przemieszczeń. Pojęcia $i$ elementy metodyki. [Basis of geodetic determinations of displacements. Notions and methodology elements] University of Technology Press, Warsaw.

Prószyński, W. (1986). Problem układu odniesienia w metodach obliczania przemieszczeń. [Datum problem in displacements analysis]. Geodesy and Cartography., 35, 47-55.

Setan, H. \& Singh R. (2001). Deformation Analysis of a Geodetic Monitoring Network. Geomatica., 55, 333-346.

Wiśniewski, Z. (1989). Estimation of local variance coefficients in adjustment of geodetic networks. Bolletino di Geodesia e Scienze Affini., 48, 165-180.

Wiśniewski, Z. (1990). Lokalne współczynniki wariancji i ich estymacja po wyrównaniu sieci geodezyjnej. [Local variance factors and their estimation after adjustment of geodetic networks]. Geodesy and Cartography., 95, 96-108. 


\section{APPENDIX 1}

$$
\begin{gathered}
\operatorname{Tr}\left(\mathbf{h}_{l} \mathbf{C}\right)=\operatorname{Tr}\left(\mathbf{F}_{l}^{T} \mathbf{P}_{l} \mathbf{F}_{l} \mathbf{C}\right)=\operatorname{Tr}\left(\mathbf{F}_{l} \mathbf{C} \mathbf{F}_{l}^{T} \mathbf{P}_{l}\right) \\
\operatorname{Tr}\left(\left(\mathbf{I}_{0 l}-\mathbf{A}_{l} \mathbf{N}^{-} \mathbf{A}^{T} \mathbf{P}\right) \mathbf{G} \mathbf{P}^{-1}\left(\mathbf{I}_{0 l}^{T}-\mathbf{P} \mathbf{A} \mathbf{N}^{-} \mathbf{A}_{l}^{T}\right) \mathbf{P}_{l}\right)= \\
\operatorname{Tr}\left(\left(\mathbf{I}_{0 l} \mathbf{G} \mathbf{P}^{-1}-\mathbf{A}_{l} \mathbf{N}^{-} \mathbf{A}^{T} \mathbf{P} \mathbf{G} \mathbf{P}^{-1}\right)\left(\mathbf{I}_{0 l}^{T}-\mathbf{P} \mathbf{A} \mathbf{N}^{-} \mathbf{A}_{l}^{T}\right) \mathbf{P}_{l}\right)= \\
\operatorname{Tr}\left(\mathbf{I}_{0 l} \mathbf{G} \mathbf{P}^{-1} \mathbf{I}_{0 l}^{T} \mathbf{P}_{l}-\mathbf{I}_{0 l} \mathbf{G A} \mathbf{N}^{-} \mathbf{A}_{l}^{T} \mathbf{P}_{l}-\mathbf{A}_{l} \mathbf{N}^{-} \mathbf{A}^{T} \mathbf{G} \mathbf{I}_{0 l}^{T} \mathbf{P}_{l}+\right. \\
\left.\mathbf{A}_{l} \mathbf{N}^{-1} \mathbf{A}^{T} \mathbf{G P A} \mathbf{N}^{-1} \mathbf{A}_{l}^{T} \mathbf{P}_{l}\right)
\end{gathered}
$$

Because

$$
\mathbf{I}_{0 l} \mathbf{G} \mathbf{P}^{-1} \mathbf{I}_{0 l}^{T} \mathbf{P}=\sigma_{0 l}^{2} \mathbf{I}_{l}, \mathbf{I}_{0 l} \mathbf{G} \mathbf{P}^{-1} \mathbf{P} \mathbf{A}=\sigma_{0 l}^{2} \mathbf{A}_{l}, \mathbf{A}^{T} \mathbf{G} \mathbf{I}_{0 l}^{T}=\sigma_{0 l}^{2} \mathbf{A}_{l}^{T}
$$

we obtain

$$
\operatorname{Tr}\left(\sigma_{0 l}^{2}\left(\mathbf{I}_{l}-\mathbf{A}_{l} \mathbf{N}^{-} \mathbf{A}_{l}^{T} \mathbf{P}_{l}-\mathbf{A}_{l} \mathbf{N}^{-} \mathbf{A}_{l}^{T} \mathbf{P}_{l}\right)+\mathbf{A}_{l} \mathbf{N}^{-} \mathbf{A}^{T} \mathbf{G} \mathbf{P} \mathbf{A} \mathbf{N}^{-} \mathbf{A}_{l}^{T} \mathbf{P}_{l}\right)
$$

Further, because

$\mathbf{A}^{T} \mathbf{G P A}=\sum_{l=1}^{r} \sigma_{0 l}^{2} \mathbf{A}_{l}^{T} \mathbf{P}_{l} \mathbf{A}_{l}$

finally, we obtain

$$
\begin{aligned}
& \operatorname{Tr}\left(\sigma_{0 l}^{2}\left(\mathbf{I}_{l}-\mathbf{A}_{l} \mathbf{N}^{-} \mathbf{A}_{l}^{T} \mathbf{P}_{l}-\mathbf{A}_{l} \mathbf{N}^{-} \mathbf{A}_{l}^{T} \mathbf{P}_{l}+\mathbf{A}_{l} \mathbf{N}^{-} \mathbf{A}_{l}^{T} \mathbf{P}_{l} \mathbf{A}_{l} \mathbf{N}^{-} \mathbf{A}_{l}^{T} \mathbf{P}_{l}\right)+\right. \\
&\left.+\sum_{k=1}^{l-1} \sigma_{0 k}^{2} \mathbf{A}_{l} \mathbf{N}^{-} \mathbf{A}_{k}^{T} \mathbf{P}_{k} \mathbf{A}_{k} \mathbf{N}^{-} \mathbf{A}_{l}^{T} \mathbf{P}_{l}+\sum_{k=l+1}^{r} \sigma_{0 k}^{2} \mathbf{A}_{l} \mathbf{N}^{-} \mathbf{A}_{k}^{T} \mathbf{P}_{k} \mathbf{A}_{k} \mathbf{N}^{-1} \mathbf{A}_{l}^{T} \mathbf{P}_{l}\right)= \\
& \operatorname{Tr}\left(\sigma_{0 l}^{2}\left(\mathbf{I}_{l}-2\left(\mathbf{A}_{l}^{T} \mathbf{P}_{l} \mathbf{A}_{l} \mathbf{N}^{-}\right)+\mathbf{A}_{l}^{T} \mathbf{P}_{l} \mathbf{A}_{l} \mathbf{N}^{-} \mathbf{A}_{l}^{T} \mathbf{P}_{l} \mathbf{A}_{l} \mathbf{N}^{-}\right)+\right. \\
&\left.+\sum_{k=1}^{l-1} \sigma_{0 k}^{2} \mathbf{A}_{l}^{T} \mathbf{P}_{l} \mathbf{A}_{l} \mathbf{N}^{-} \mathbf{A}_{k}^{T} \mathbf{P}_{k} \mathbf{A}_{k} \mathbf{N}^{-}+\sum_{k=l+1}^{r} \sigma_{0 k}^{2} \mathbf{A}_{l}^{T} \mathbf{P}_{l} \mathbf{A}_{l} \mathbf{N}^{-} \mathbf{A}_{k}^{T} \mathbf{P}_{k} \mathbf{A}_{k} \mathbf{N}^{-}\right)
\end{aligned}
$$


Statystyczna istotność przemieszczeń w niejednorodnych sieciach kontrolnych

\title{
Krzysztof Nowel, Waldemar Kamiński
}

\author{
Uniwersytet Warmińsko-Mazurski \\ Wydział Geodezji i Gospodarki Przestrzennej \\ Instytut Geodezji \\ ul. Oczapowskiego 1., 10-719 Olsztyn, Polska \\ e-mail: krzysztof.nowel@uwm.edu.pl,waldemar.kaminski@uwm.edu.pl
}

\section{Streszczenie}

W pracy zaproponowano modyfikację klasycznego procesu oceny statystycznej istotności przemieszczeń w przypadku niejednorodnych sieci kontrolnych (np. kątowo- liniowych) zakładanych do pomiarów i analizy deformacji. Podstawą proponowanego rozwiązania jest idea lokalnych współczynników wariancji. Rozważania teoretyczne uzupełniono przykładem zastosowania na symulowanej poziomej sieci kontrolnej. Uzyskane wyniki pokazały, że ocena statystycznej istotności przemieszczeń w przypadku niejednorodnych sieci kontrolnych powinna być przeprowadzana z użyciem właśnie estymatorów lokalnych współczynników wariancji. 